\title{
The relationship between uterine corpus inclination and the outcomes of in vitro fertilization and embryo transfer: a retrospective study
}

\author{
Xiuxia Wang ${ }^{1}$, Na Zuo ${ }^{1}$, Ning-Ning Zhang ${ }^{2}$, Sitong Dong ${ }^{1}$, Wei Zhang ${ }^{1}$, and Da Li $^{2}$ \\ ${ }^{1}$ Affiliation not available \\ ${ }^{2}$ Shengjing Hospital of China Medical University
}

June 24, 2021

\begin{abstract}
Objective: To investigate the impact of the uterine corpus inclination on pregnancy outcomes in in vitro fertilization and embryo transfer (IVF-ET). Design: Retrospective cohort study. Setting: University-based reproductive medicine center. Population: 526 patients underwent their first frozen embryo transfer (FET) cycle. Methods: All patients underwent transvaginal ultrasound examination to measure the distance from the midline of uterine cavity to the ultrasound probe, which indirectly reflect the uterine corpus inclination. The uterine corpus tends toward the horizontal position as the average distance increases. Multivariable regression analysis was used to study the effect of the uterine corpus inclination on pregnancy outcomes, even in different embryo stage cohorts as subgroup analysis. Main outcomes measures: Clinical pregnancy rate (CPR), spontaneous abortion rate, and live birth rate (LBR). Results:The patients were grouped according to the quartiles of the average distance distribution (Group 1, [?] $1.98 \mathrm{~cm}$; Group 2, 1.99-2.19 cm; Group 3, 2.20-2.50 cm; Group 4, [?] $2.51 \mathrm{~cm}$ ). There were statistically significant differences in the CPR and LBR between Groups 1 and 4 in the cleavage-stage ET cohort (CPR: adjusted odds ratio [OR] 0.225, 95\% confidence interval [CI] 0.068-0.774; LBR: adjusted OR 0.315, 95\% CI 0.100-0.996) $(\mathrm{P}<0.05)$. There were no significant differences among the four groups in pregnancy outcomes in the blastocyst transfer cohort $(\mathrm{P}>0.05)$. The cut-off value of $2.146 \mathrm{~cm}$ was calculated to predict the pregnancy outcomes in the cleavage-stage ET cohort. Conclusion:The uterine corpus inclination might be an independent risk factor for the success of cleavage-stage ET
\end{abstract}

\section{Introduction}

In vitro fertilization and embryo transfer (IVF-ET) is a globally utilized form of assisted reproductive technology, and ET is the final and most essential step thereof ${ }^{1}$. During the ET process, the fertilized embryos are cultured in vitro and placed directly inside the uterine lumen via an ET catheter ${ }^{2}$. Previous studies investigating the main factors affecting the implantation rate reported endometrial receptivity and embryo quality as risk factors ${ }^{3}$. However, ET, as the last and probably most underestimated step, has not been developed as much as the other aspects of the IVF process. Studies have shown that transferred embryos do not always implant in situ, despite the transfer process being performed under ultrasound guidance ${ }^{4,5}$. The catheter loading, catheter position, injection speed, uterine peristalsis and patients' posture during ET may affect the migration of embryos ${ }^{4,6,7}$. Poindexter et al. reported that approximately $15 \%$ of the transferred embryos were expelled from the uterine cavity and were found in the cervical os, fallopian tubes, or the ET catheter ${ }^{8}$. Baba demonstrated that approximately $80 \%$ of embryos implant in areas to which they initially are transferred and approximately $20 \%$ implant in other areas ${ }^{9}$. The migration of the transferred embryos might make detrimental effect on a successful pregnancy, and also predisposes the patient to ectopic pregnancy ${ }^{10}$.

A laboratory simulation of ET into a uterine model demonstrated that the dispersion of the transferred 
matter depend on the position of sagittal cross-section of the uterine cavity with respect to the horizon ${ }^{6}$. However, the in vitro model can not represent the real situation in vivo, and the cleavage stage embryos and blastocysts might be affected differently by the inclination of uterus due to their different migration duration before implantation. Therefore, it remains unclear whether the inclination of uterine corpus affects the pregnancy outcomes of IVF-ET.

This study investigated whether the inclination of the uterine corpus affect the pregnancy outcomes in IVF-ET, and whether different uterine inclination affect the pregnancy outcomes of embryos at different developmental stages.

\section{Material and methods}

\section{Study population}

This was a retrospective study of patients who received IVE-ET treatment from June 2018 to December 2019 at our reproductive medicine center and the study was conducted in accordance with ethical standards of China Medical University (2021PS014F). We included patients who (1) were undergoing their first FET cycle due to failure in their fresh ET attempt or adopting a freeze-all strategy in their fresh cycle, (2) were aged 20-45 years, (3) one or two embryos of good quality or higher for transfer (embryos were evaluated for cleavage on day 3 based on the Istanbul consensus ${ }^{11}$, and blastocyst morphology was evaluated according to the Gardner criteria ${ }^{12}$ ), (4) underwent endometrial preparation with hormone replacement therapy (HRT) in the FET cycles, (5) uterine length 70-80 mm and uterine anteroposterior diameter 20-30mm, (6) the endometrial thickness on ET day was more than $8 \mathrm{~mm}$, and (7) had a uterus in the "anteverted and anteflexed", "retroverted and retroflexed", and "horizontal" positions.

The exclusion criteria were as follows: (1) uterine abnormalities, (2) endometriosis or adenomyosis, (3) endometrial polyps or uterine myomas, (4) autoimmune, endocrine, or metabolic diseases, (5) previous diagnosis of uterine adhesion, (6) history of recurrent miscarriage, (7) chromosomal abnormalities, (8) undergoing blastocyst biopsy for pre-implantation genetic testing, (9) the endometrial thickness were less than $8 \mathrm{~mm}$ on ET day, (10) uterus in the "anteverted and retroflexed" or "retroverted and anteflexed" position, (11) missing data, and (12) ectopic pregnancy as pregnancy outcome. Total number of patients before inclusion criteria were 1703. According to inclusion and exclusion criteria, 526 patients who underwent their first FET cycle were enrolled (Figure S1). Among them, 35 patients failed in their fresh ET attempts and 491 patients adopted a freeze-all strategy in their fresh IVF cycles. The overall demographic data, IVF characteristics, and pregnancy outcomes of the whole study population are detailed in Table 1.

Ovarian stimulation protocol, endometrial preparation protocol, ET process, and luteal support

A personalized controlled ovarian hyperstimulation $(\mathrm{COH})$ protocol was chosen for each patient according to her age, anti-Müllerian hormone level, body mass index (BMI), number of antral follicles in the bilateral ovaries, and prior response to stimulation ${ }^{13-15}$. Follicular development was determined by transvaginal ultrasonography, and the dosages of gonadotropin were adjusted according to different ovarian responses.

When the follicles reached a diameter of more than $17 \mathrm{~mm}$, the trigger was performed for final oocyte maturation. Oocyte retrieval was performed 36 hours after triggering with transvaginal ultrasound-guided aspiration.

Endometrial preparation for FET used the HRT cycle, wherein 4-8 $\mathrm{mg}$ of estradiol valerate (Progynova, Bayer, Germany) was administered orally for at least 10 days from the 3rd to 5 th days of menstruation to promote endometrial growth. The cut-off value for endometrial transformation was more than $8 \mathrm{~mm}$. The embryos were slowly injected $10 \mathrm{~mm}$ from the uterine fundus under ultrasound guidance, and the catheter remained in situ for 3-5 seconds. The patients subsequently stood upright and walked to the rest area, where they lay in bed for 20-30 min before leaving.

The luteal phase was supported by vaginal administration of progesterone gel (Crinone, Fleet Laboratories 
Ltd., UK) at $90 \mathrm{mg} /$ day, while estradiol was maintained at the original dose. Luteal support was continued until 11 weeks of gestational age.

\section{Ultrasound measurement of the uterus}

Transvaginal ultrasound measurements were performed on the day of ET, using a Voluson E8 (GE, Healthcare Austria $\mathrm{GmbH}$ ) equipped with a $46-\mathrm{Hz}$ endovaginal transducer, at $165^{\circ}$. The patients were guided to empty the bladder and lie supine on the examination table, which was inclined at $30^{\circ}$, in the lithotomy position. The entire uterine corpus needed to be seen on a longitudinal transvaginal grayscale image, and the ultrasound probe was placed as close to the uterus corpus as possible without abdominal compression to ensure a clear image. In order to obtain a clear ultrasound image, we do not guarantee that the ultrasound probe is horizontal, so the respective ultrasound images we obtained from ultrasonography can not objectively reflect the inclination angle of the uterine corpus. So we used the vertical distance from the midline of uterine cavity to the ultrasonic probe to indirectly reflect the inclination degree of uterine corpus. An example of the calculated average distance was shown in Figure 1b, where the midline of the uterine cavity (from the anatomic internal cervical os to the fundal endometrium) was divided into four equal parts and the vertical distances were measured from each split point to the ultrasound probe (five lines in total); the average distance was calculated according to the average of the sum of the five lines. The schematic in Figure 1 illustrated the smaller average distance from the midline of uterine corpus to the ultrasonic probe, the greater inclination of the uterine corpus was. Conversely, the greater average length from the midline of uterine cavity to the ultrasound probe, the more the uterine corpus tended toward the horizontal position. All measurements were performed by two experienced doctors.

\section{Statistical analysis}

The pregnancy outcomes in our study included the clinical pregnancy rate (CPR), spontaneous abortion rate (SAR), and live birth rate (LBR). Clinical pregnancy was defined as detection of a gestational sac on ultrasound at 7 gestational weeks ${ }^{16}$. In China, spontaneous abortion is defined as the spontaneous loss of a clinical pregnancy $<28$ gestational weeks, and live birth as the survival of delivery [?] 28 gestational weeks.

The patients were divided into four groups according to the 1st, 2nd, and 3rd quartiles of the overall average distance distribution, which represent different uterine corpus inclinations (Group 1, [?] $1.98 \mathrm{~cm}, \mathrm{n}=131$; Group 2, 1.99-2.19 cm, n = 133; Group 3, 2.20-2.50 cm, n =130; Group 4, [?] $2.51 \mathrm{~cm}, \mathrm{n}=129$ ).

Multivariable logistic regression models were calculated for the whole cohort, with the uterine corpus inclination as the main variable of interest. The potential risk factors entered into the multivariable regression model were those that showed clinical relevance or those with a $P$ value $<0.2$ on univariate analysis. The included variables were carefully selected based on the number of events available to ensure the stability of the regression equation. Adjusted odds ratios (OR) and their 95\% confidence intervals (CI) were calculated to analyze the independent effect of the uterine corpus inclination on the pregnancy outcomes. As the migratory duration before implantation differed between cleavage-stage embryos and blastocysts, we analyzed the two stages separately. The number of cleavage ET cohort was 126, and the number of blastocyst transfer cohort was 400.

The second aim of the study was to determine the cut-off point for the average distance that would predict pregnancy outcomes. Receiver operating characteristic (ROC) curves were used to estimate the sensitivity and specificity of the average distance for predicting pregnancy outcomes. $P<0.05$ was considered to indicate statistical significance. All statistical analyses were performed using IBM SPSS 26.0 (IBM Corp., Armonk, NY, USA).

\section{Results}

The calculated distance distribution of the whole cohort ranged from 1.410 to $3.962 \mathrm{~cm}$. The average distance ranges of the four groups were as follows: Group 1, [?] $1.98 \mathrm{~cm}$; Group 2, 1.99-2.19 cm; Group 3, 2.20-2.50 cm; Group 4, [?] $2.51 \mathrm{~cm}$, which represented different inclination degrees of uterine corpus. The description 
of pregnancy outcomes of the entire cohort, cleavage-stage ET cohort, and blastocyst transfer cohort in the different inclination groups are detailed in Figure S2.

Multivariable logistic regression analysis on uterine corpus inclination and potential risk factors for pregnancy outcomes in the entire cohort

Multivariable logistic regression analysis of the entire cohort demonstrated that there was a statistically significant difference in CPR between Groups 4 and 1 [adjusted OR 0.501, 95\% CI 0.292-0.859 $(P<0.05)$ ], which adjusted for maternal age, BMI, insemination method, outcomes of fresh cycle, number of embryos transferred, embryo stage, and causes of infertility. There were no statistically significant differences in the CPR between Groups 2 and 1 (adjusted OR 0.801,95\% CI 0.465-1.379) or Groups 3 and 1 (adjusted OR $0.806,95 \%$ CI 0.463-1.403). No statistically significant differences were found on SARs (adjusted for maternal age and BMI) and on LBRs (adjusted for maternal age, BMI, insemination method, outcomes of fresh cycle, number of embryos transferred, embryo stage, and causes of infertility) between the four groups $(P>0.05)$ (Table S1).

Multivariable logistic regression analysis on uterine corpus inclination and potential risk factors for pregnancy outcomes in the cleavage-stage ET cohort

Multivariable logistic regression analysis on the cleavage-stage ET cohort demonstrated that there were statistically significant differences in CPR and LBR between Group 4 and Group 1 (CPR, adjusted OR, 0.225, 95\% CI, 0.068-0.774; LBR, adjusted OR, 0.315, 95\% CI, 0.100-0.996) $(P<0.05)$, when adjusted for maternal age, BMI, number of embryos transferred, embryo stage, and infertility cause of ovulatory disorders and male factors. No statistically significant differences were found in the CPRs and LBRs between Groups 2 and 1 or Groups 3 and $1(P>0.05)$ (Table 2$)$.

Multivariable logistic regression analysis on uterine corpus inclination and potential risk factors for pregnancy outcomes in the blastocyst transfer cohort

Multivariable logistic regression analysis demonstrated that the inclination of the uterine corpus was not an independent risk factor for pregnancy outcomes in the blastocyst transfer cohort $(P>0.05)$ (Table 3).

\section{ROC analyses on the cleavage-stage ET cohort}

ROC analysis was performed on the cleavage-stage ET cohort, and the ROC curve for the average distance between the midline of the uterine cavity and the ultrasound probe indicated that the cut-off value of 2.146 $\mathrm{cm}$ showed $77.2 \%$ sensitivity and $52.2 \%$ specificity in predicting clinical pregnancy, with an area under the ROC curve of $0.667(P=0.001)$. Additionally, the cut-off value of $2.146 \mathrm{~cm}$ showed $72.1 \%$ sensitivity and $51.7 \%$ specificity in predicting live birth, with an area under the ROC curve of $0.632(P=0.011)$ (Figure S3).

Regrouping based on the cut-off value to investigate whether embryo stages were an independent risk factor for pregnancy outcomes

Multivariable logistic regression analysis on the cohort with an average distance under $2.146 \mathrm{~cm}$ demonstrated no statistically significant differences in the CPR and LBR between the blastocyst transfer and cleavage-stage ET groups (CPR: adjusted OR 2.165, 95\% CI 0.284-16.494; LBR: adjusted OR 1.494, 95\% CI 0.225-9.908; $P>0.05$ ), which were adjusted for maternal age, BMI, outcomes of the $\mathrm{COH}$ cycle, insemination method, number of embryos transferred, and infertility causes.

Multivariable logistic regression analysis on the cohort with an average distance [?] $2.146 \mathrm{~cm}$ demonstrated that the CRP and LBR of the blastocyst transfer group were significantly higher than those of the cleavagestage ET group (CPR: adjusted OR 9.834, 95\% CI 2.035-47.526; LBR: adjusted OR 4.135, 95\% CI 1.06316.083) $(P<0.05)$ (Tables S2, S3).

\section{Discussion}

Main findings: 
This study showed that the inclination of uterine corpus might be an independent risk factor for the success of cleavage stage embryo transfer population. The more the uterine corpus tended to horizontal position, the lower the CPR and LBR of the cleavage-stage ET group. However, blastocyst transfer were not affected by the uterine corpus inclination. Moreover, there were no significant difference in pregnancy outcomes between cleavage stage embryo transfer and blastocyst transfer when the inclination of uterus was larger; when the uterus tends to the horizontal position (small uterus inclination ), the CPR and LBR of blastocyst transfer group were significantly higher than that of cleavage stage embryo transfer group. These findings suggest that active blastocyst transfer should be advised for the uterus tending toward the horizontal position, avoiding the influence of uterine position on pregnancy outcomes; for the patients with a uterus with greater inclination who have fewer embryos, blastocyst transfer should be avoided because of its non-superiority, to prevent the loss of precious embryos in the cultivation process ${ }^{17}$.

\section{Strengths and limitations}

To our knowledge this is the first cohort study to investigate the relationship between uterine corpus inclination and pregnancy outcomes of IVF-ET. To avoid the influence of $\mathrm{COH}$ on the endometrial receptivity and the interference of cumulative pregnancy rate, we only selected patients undergoing their first FET cycle and who used HRT for endometrial preparation. We excluded cases with adenomyosis, myoma, and "anteverted and retroflexed" and "retroverted and anteflexed" uteri, and limited size of uterus in the included patients, because these gynecological diseases and the associated cervical distortion would increase the distance between the midline of uterine cavity and the ultrasonic probe, which would not objectively reflect the inclination of the uterine corpus in our calculated method.

The main limitation of this study lies in the method of measuring the uterine corpus inclination. In order to get a clear image, we would rotate the ultrasound probe to make the ultrasound probe as close as possible to the uterine corpus, and we couldn't guarantee that the ultrasonic probe kept horizontal when we did the ultrasound examination. Therefore, we couldn't get the actual uterine inclination angle in our retrospective ultrasound images. A great quantity of clinical work in our experience we found that the more horizontal the uterus, the farther the distance from the uterine cavity to the ultrasound probe, so we used the average distance from the midline of uterine cavity to the ultrasound probe to indirectly reflect the uterine corpus inclination. We tried to use the average distance of three, five, and eleven lines to represent the distance, and we found that the average distance of five lines and eleven lines gave very similar results, while the average values of three lines differ. We therefore selected five lines to represent the distance to simplify our measurement. Although we did not give an accurate method to measure the tilt angle of uterine corpus, this measurement method could be applied to our retrospective images, and it could also truly reflect the uterine inclination degree. Other limitations of this study lies in its retrospective nature and the possibility of unmeasured confounding factors, such as smoking habits and alcohol consumption.

\section{Interpretation}

The natural position of the uterus depends on the uterine ligament and the pelvic floor muscles and fascia. It affects the incidence of some gynecological diseases and postoperative complications. A study in 1987 reported that retroversion of uterus was positively correlated with the occurrence of endometriosis ${ }^{18}$. Ante-version and retroversion of uterus are more likely to suffer from chronic pelvic pain, dyspareunia and dysmenorrhea 19 , and a higher angle of flexion was associated with increased menstrual pain ${ }^{20}$. Cesarean section scar diverticulum is more prevalent in patients with a retro-flexed uterus than in those with an ante-flexed uterus 21. Retroversion of uterus was significantly associated with occurrence of intraoperative complications such as uterine perforation during curettage ${ }^{22,23}$. The pathogenesis of all these diseases is related to the physical aspect and anatomical characteristics of the uterus. IVF is a non-physiological process and ET is an human operated invasive procedure, we believe that the uterine corpus inclination might be an independent risk factor for implantation of the cleavage-stage embryos.

Our results demonstrated that the more the uterine corpus tends to horizontal position, the lower the CPR and LBR of the cleavage-stage ET group. This result is in consist with the conclusion of laboratory model in 
vitro which demonstrated that the transfer liquid is more likely to be pulled backward toward the cervix in the uterus with horizontal position, which may make adverse effect on pregnancy rate ${ }^{6}$. However, the CPR and LBR of the blastocyst transfer cohort were not affected by the inclination of uterus in this study. We speculate that it may be related to the different migration duration of the two kinds of embryos before implantation. The cleavage-stage embryos need to migrate for 3-4 days before implantation ${ }^{6}$, whereas the blastocysts implant immediately after deposition ${ }^{24}$. We hypothesized that the longer the embryo migration duration, the more likely the embryo migration trajectory was affected by the reduced inclination of the uterine corpus; moreover, the grafts might flow downward under the influence of gravity in a less inclined uterus, so that the embryos might leave the optimal implantation position and affect pregnancy outcomes. In addition, this study demonstrated that the pregnancy outcomes were not statistically difference between anteverted uteri and retroverted uteri (OR 1.030, 95\% CI 0.677-1.568), which was consistent with the prospective study conducted by Egbase et al. ${ }^{25}$.

Our results contradict the previous understanding that the pregnancy rate of blastocyst transfer is always higher than that of cleavage-stage ET ${ }^{26}$. We found no significant difference in the CPR, SAR and LBR between the cleavage-stage ET and blastocyst transfer cohorts when the distance was under $2.146 \mathrm{~cm}$ (which represent a more tiled uterus). In contrast, when the distance exceeded $2.146 \mathrm{~cm}$ (which represent a uterus more tend to horizontal position), the CPR and LBR of the blastocyst transfer cohort was significantly higher than that of the cleavage-stage ET cohort. This indicates that active blastocyst transfer should be advised for patients with a uterus tending toward the horizontal position, avoiding the influence of uterine position on pregnancy outcomes. For the patients with a uterus with greater inclination and fewer embryos, blastocyst transfer should be avoided because of its non-superiority, to prevent the loss of precious embryos in the cultivation process ${ }^{17}$..

This study has made a potential contribution to the effect of uterine corpus inclination on embryo implantation in IVF-ET. A multicenter randomized controlled trial with a more accurate measurements are needed to further confirm this conclusion, and that is what we are doing right now.

\section{Conclusions}

The inclination of uterine corpus might affect the pregnancy outcomes of IVF-ET. Individual embryo transfer protocol for different uterus anatomy characteristics is recommended for improving this technology.

\section{Authors' roles}

X.X.W. conceived and designed the study. N.Z., N.N.Z., W.Z. performed the data collection. N.Z., D.L. and N.N.Z. performed the statistical analysis. Sitong Dong performed the figure processing. X.X.W., D.L. and N.Z. wrote the paper. All authors approved the final manuscript.

\section{Conflict of interest}

The authors declare no conflict of interest.

\section{References}

1. Cozzolino M, Busnelli A, Pellegrini L, Riviello E, Vitagliano A. How vitamin D level influences in vitro fertilization outcomes: results of a systematic review and meta-analysis. Fertil Steril. 2020 Nov;114(5):101425.

2. Li Z, Sullivan EA, Chapman M, Farquhar C, Wang YA. Risk of ectopic pregnancy lowest with transfer of single frozen blastocyst. Hum Reprod. 2015 Sep;30(9):2048-54.

3. Templeton A, Morris JK, Parslow W. Factors that affect outcome of in-vitro fertilisation treatment. Lancet. 1996 Nov 23;348(9039):1402-6.

4. Knutzen V, Stratton CJ, Sher G, McNamee PI, Huang TT, Soto-Albors C. Mock embryo transfer in early luteal phase, the cycle before in vitro fertilization and embryo transfer: a descriptive study. Fertil Steril. 1992 Jan;57(1):156-62. 
5. Mansour RT, Aboulghar MA, Serour GI, Amin YM. Dummy embryo transfer using methylene blue dye. Hum Reprod. 1994 Jul;9(7):1257-9.

6. Eytan O, Elad D, Jaffa AJ. Evaluation of the embryo transfer protocol by a laboratory model of the uterus. Fertil Steril. 2007 Aug;88(2):485-93.

7. Zhu L, Xiao L, Che HS, Li YP, Liao JT. Uterine peristalsis exerts control over fluid migration after mock embryo transfer. Hum Reprod. 2014 Feb;29(2):279-85.

8. Poindexter AN, 3rd, Thompson DJ, Gibbons WE, Findley WE, Dodson MG, Young RL. Residual embryos in failed embryo transfer. Fertil Steril. 1986 Aug;46(2):262-7.

9. Baba K, Ishihara O, Hayashi N, Saitoh M, Taya J, Kinoshita K. Where does the embryo implant after embryo transfer in humans? Fertil Steril. 2000 Jan;73(1):123-5.

10. Saravelos SH, Balfoussia DT, Kong G, Chung J, Mak J, Chung C, et al. Embryo migration following ART documented by 2D/3D ultrasound. Facts Views Vis Obgyn. 2020 Aug 5;12(2):143-8.

11. Embryology. ASiRMaESIGo. The Istanbul consensus workshop on embryo assessment: proceedings of an expert meeting. Hum Reprod. 2011 Jun;26(6):1270-83.

12. Gardner DK, Surrey E, Minjarez D, Leitz A, Stevens J, Schoolcraft WB. Single blastocyst transfer: a prospective randomized trial. Fertil Steril. 2004 Mar;81(3):551-5.

13. Maher JY, Christianson MS. Controlled ovarian stimulation and triggers in in vitro fertilization: protocol personalization key to optimize outcomes. Minerva Endocrinol. 2018 Mar;43(1):37-49.

14. Al-Inany HG, Youssef MA, Ayeleke RO, Brown J, Lam WS, Broekmans FJ. Gonadotrophin-releasing hormone antagonists for assisted reproductive technology. Cochrane Database Syst Rev. 2016 Apr 29;4:CD001750.

15. Siristatidis CS, Gibreel A, Basios G, Maheshwari A, Bhattacharya S. Gonadotrophin-releasing hormone agonist protocols for pituitary suppression in assisted reproduction. Cochrane Database Syst Rev. 2015 Nov 9(11):CD006919.

16. Zegers-Hochschild F, Adamson GD, de Mouzon J, Ishihara O, Mansour R, Nygren K, et al. International Committee for Monitoring Assisted Reproductive Technology (ICMART) and the World Health Organization (WHO) revised glossary of ART terminology, 2009. Fertil Steril. 2009 Nov;92(5):1520-4.

17. Glujovsky D, Farquhar C. Cleavage-stage or blastocyst transfer: what are the benefits and harms? Fertil Steril. 2016 Aug;106(2):244-50.

18. Moen MH. Endometriosis in women at interval sterilization. Acta Obstet Gynecol Scand. 1987;66(5):4514 .

19. Fauconnier A, Dubuisson JB, Foulot H, Deyrolles C, Sarrot F, Laveyssiere MN, et al. Mobile uterine retroversion is associated with dyspareunia and dysmenorrhea in an unselected population of women. Eur J Obstet Gynecol Reprod Biol. 2006 Aug;127(2):252-6.

20. Cagnacci A, Grandi G, Cannoletta M, Xholli A, Piacenti I, Volpe A. Intensity of menstrual pain and estimated angle of uterine flexion. Acta Obstet Gynecol Scand. 2014 Jan;93(1):58-63.

21. Raimondo G, Grifone G, Raimondo D, Seracchioli R, Scambia G, Masciullo V. Hysteroscopic treatment of symptomatic cesarean-induced isthmocele: a prospective study. J Minim Invasive Gynecol. 2015 Feb;22(2):297-301.

22. Hefler L, Lemach A, Seebacher V, Polterauer S, Tempfer C, Reinthaller A. The intraoperative complication rate of nonobstetric dilation and curettage. Obstet Gynecol. 2009 Jun;113(6):1268-71. 
23. Nathanson BN. Management of uterine perforations suffered at elective abortion. Am J Obstet Gynecol. 1972 Dec 15;114(8):1054-9.

24. Milki AA, Jun SH. Ectopic pregnancy rates with day 3 versus day 5 embryo transfer: a retrospective analysis. BMC Pregnancy Childbirth. 2003 Nov 7;3(1):7.

25. Egbase PE, Al-Sharhan M, Grudzinskas JG. Influence of position and length of uterus on implantation and clinical pregnancy rates in IVF and embryo transfer treatment cycles. Hum Reprod. 2000 Sep;15(9):1943-6.

26. Holden EC, Kashani BN, Morelli SS, Alderson D, Jindal SK, Ohman-Strickland PA, et al. Improved outcomes after blastocyst-stage frozen-thawed embryo transfers compared with cleavage stage: a Society for Assisted Reproductive Technologies Clinical Outcomes Reporting System study. Fertil Steril. 2018 Jul 1;110(1):89-94 e2.

\section{Hosted file}

Table 1.docx available at https://authorea.com/users/421670/articles/527607-the-relationshipbetween-uterine-corpus-inclination-and-the-outcomes-of-in-vitro-fertilization-andembryo-transfer-a-retrospective-study

\section{Hosted file}

Table 2.docx available at https://authorea.com/users/421670/articles/527607-the-relationshipbetween-uterine-corpus-inclination-and-the-outcomes-of-in-vitro-fertilization-andembryo-transfer-a-retrospective-study

\section{Hosted file}

Table 3.docx available at https://authorea.com/users/421670/articles/527607-the-relationshipbetween-uterine-corpus-inclination-and-the-outcomes-of-in-vitro-fertilization-andembryo-transfer-a-retrospective-study

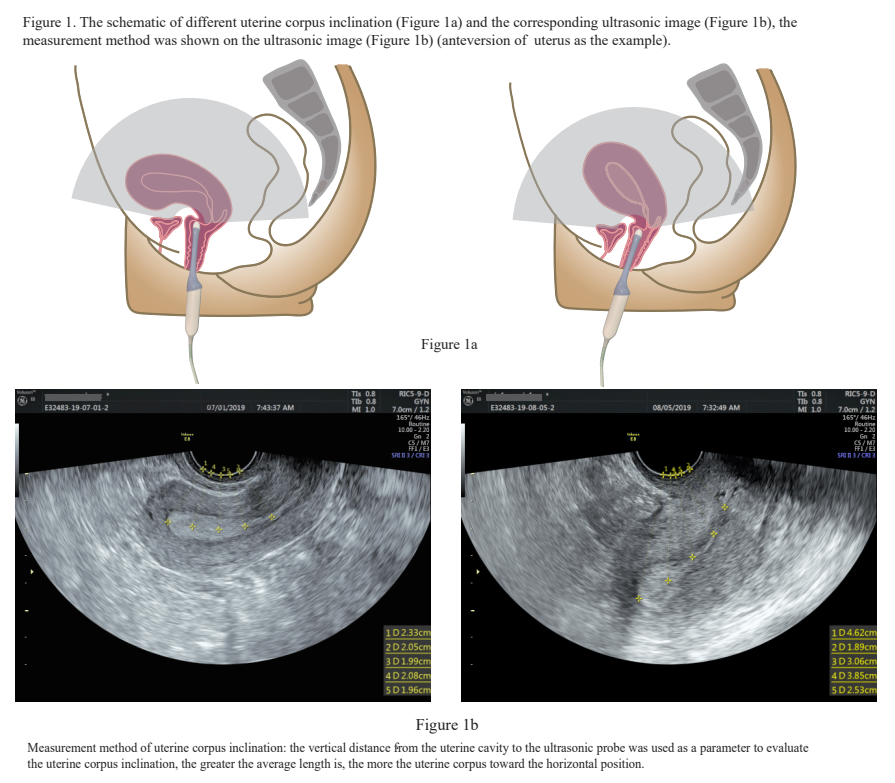

